\title{
HabEx Telescope WFE stability specification derived from coronagraph starlight leakage
}

\author{
Bijan Nemati (UAH) \\ H. Philip Stahl (MSFC) \\ Mark Stahl (MSFC) \\ SPIE Optics + Photonics \\ San Diego, CA, August 23, 2018
}




\section{Executive Summary \#1}

- Exoplanet Science is hard. It requires that the telescope and coronagraph be designed as an integrated system.

- We describe a rigorous systems engineering methodology for deriving telescope performance specifications from coronagraph performance based on a raw contrast stability error budget.

- To illustrate the methodology, we apply it to four different architectures:

1. 4-m Off-Axis Unobscured Monolithic Circular Aperture with VVC-4 Coronagraph

2. 4-m Off-Axis Unobscured Monolithic Circular Aperture with VVC-6 Coronagraph

3. 4-m Off-Axis Unobscured Monolithic Circular Aperture with HLC Coronagraph

4. 6-m On-Axis Obscured Hex Segmented Aperture with APLC Coronagraph

- HabEx Baseline (4-m Monolith - VVC-6) has the best performance.

- Architecture 4 (6-m Segmented - APLC) has the worst total performance. 


\section{Executive Summary \#2}

\section{Telescope Wavefront Stability Tolerances for 4 Coronagraphs:}

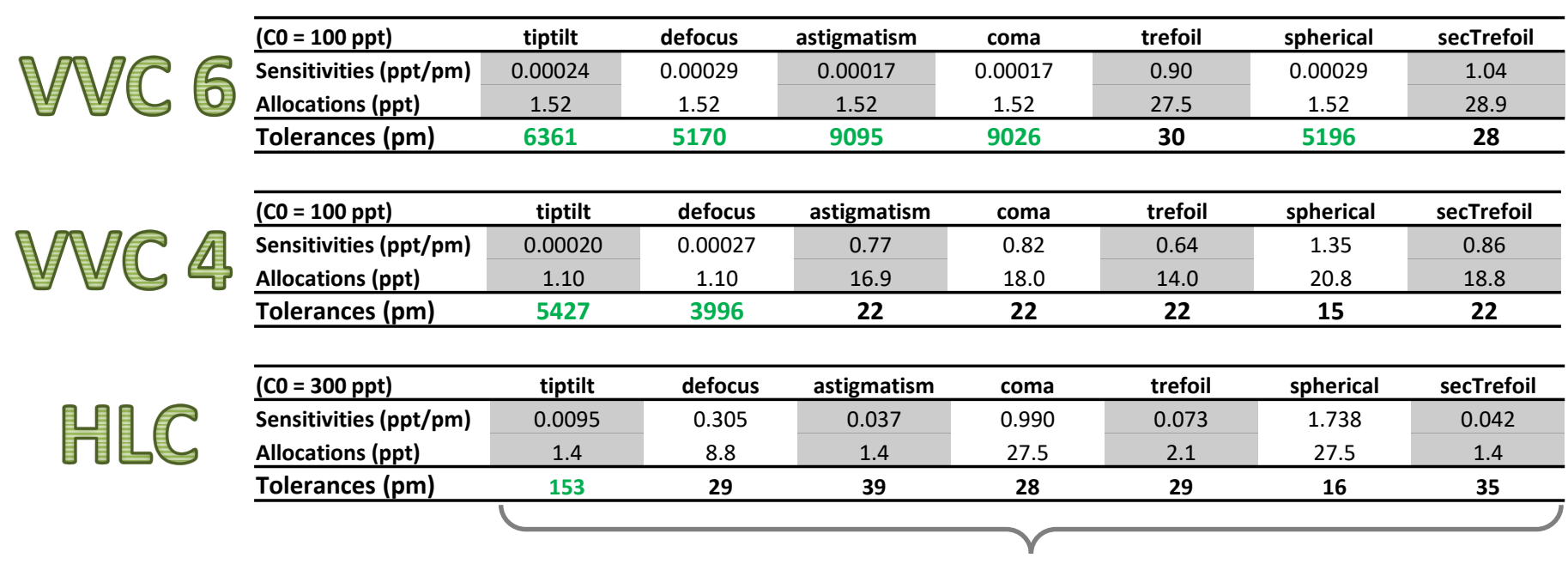

peak to valley

\begin{tabular}{|c|c|c|c|c|c|c|c|c|c|c|c|c|c|}
\hline$(C 0=100 \mathrm{ppt})$ & g_bend & g_powerS & g_spherS & g_comaS & g_comaZ & g_trefZ & g_hexfZ & s_piston & s_tiptilt & s_powerS & s_astigZ & s_trefZ & s_hexfZ \\
\hline Sensitivities (ppt/pm) & 0.15 & 0.21 & 0.090 & 0.59 & 1.32 & 0.89 & 0.12 & 5.53 & 3.26 & 1.44 & 1.71 & 1.14 & 0.15 \\
\hline Allocations (ppt) & 2.3 & 3.3 & 1.4 & 9.1 & 14.7 & 13.8 & 1.9 & 14.7 & 14.7 & 14.7 & 14.7 & 14.7 & 2.3 \\
\hline
\end{tabular}




\section{Introduction: HabEx and its Coronagraph}

HabEx is a space-based 4-meter diameter telescope with ultraviolet (UV), optical, and near-infrared (near-IR) imaging and spectroscopy capabilities.

Three driving science goals during its five-year primary mission:

To seek out nearby worlds and explore their habitability.

To map out nearby planetary systems and understand the diversity of the worlds they contain.

To carry out observations that open up new windows on the universe from the UV through

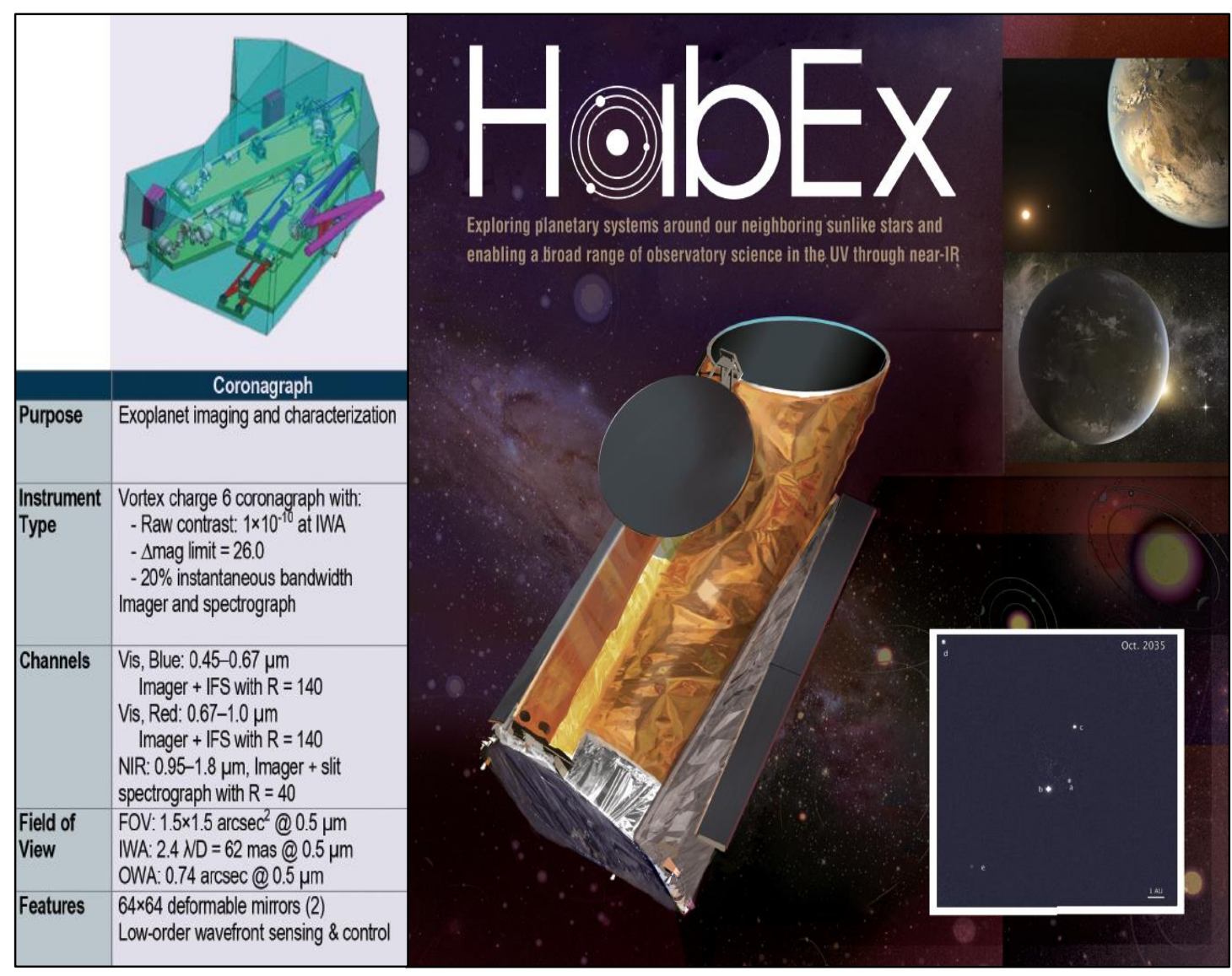
near-IR. 


\section{What does it take to see an exo-Earth?}

The flux ratio of the earth, relative to the sun, is $\sim 2.1 \times 10^{-10}$

\section{(210 ppt)}

If we could look at our solar system

- in the visible band

- from 10 pc away

- using a 4-meter telescope

- without coronagraph, the Earth would be buried under the third airy ring of the sun,

by a factor of $>5$ million

- Need to divert diffracted starlight

- We do this with a coronagraph
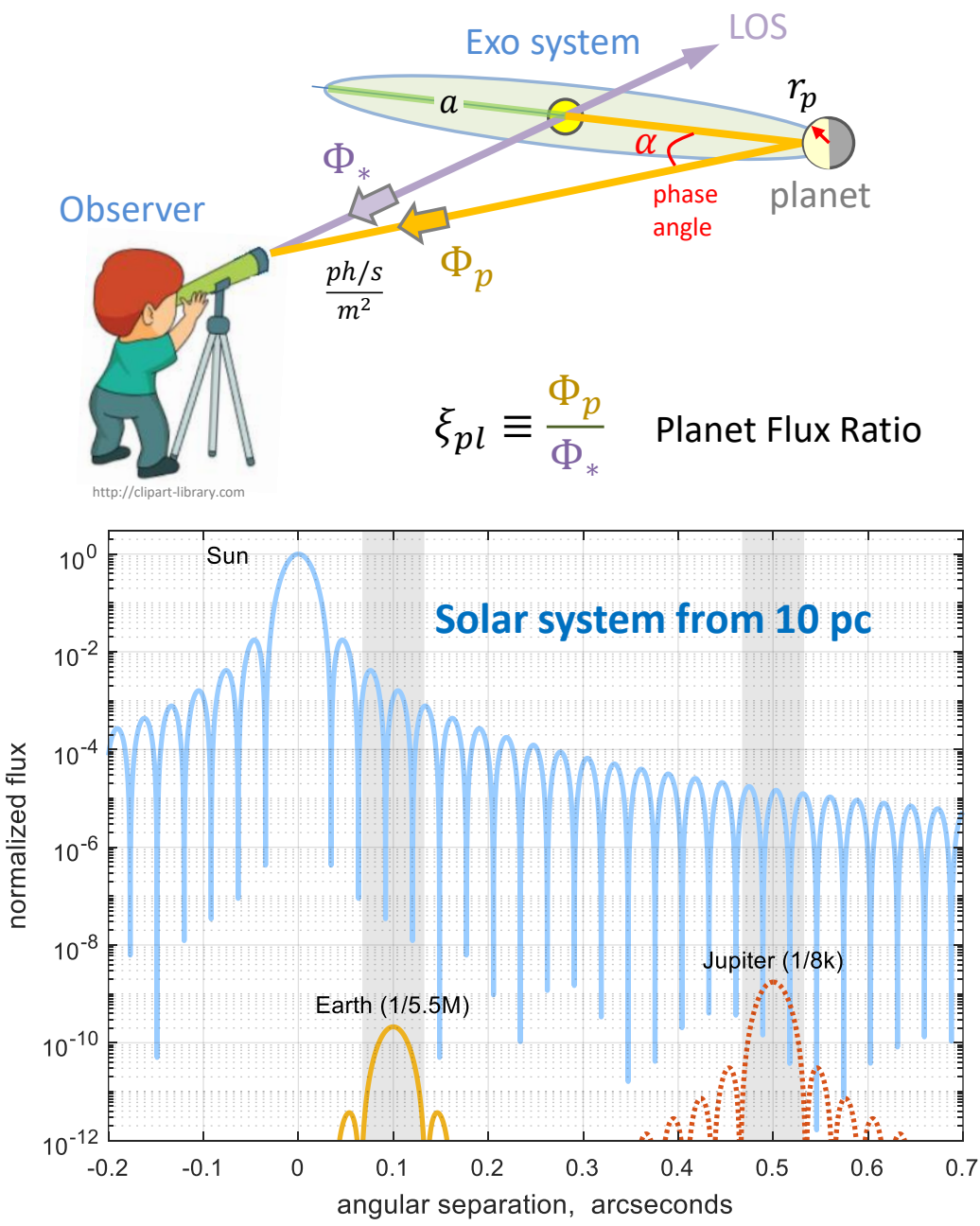


\section{Coronagraph Elements}

- Coronagraph suppresses starlight to allow detection of planet.

- Control diffraction by manipulating the phase and amplitude at a number of planes. Typically via 3 masks and 1-2 deformable mirrors.

- Result is a Dark Hole within which starlight is suppressed strongly relative to planet light.

- Inner and outer working angles are radial limits of a dark hole:

- IWA is the angle below which the planet light throughput drops to $<0.5$ of its peak value within the dark hole.

- OWA the maximum angle where starlight suppression occurs, limited by the number of deformable mirror actuators.
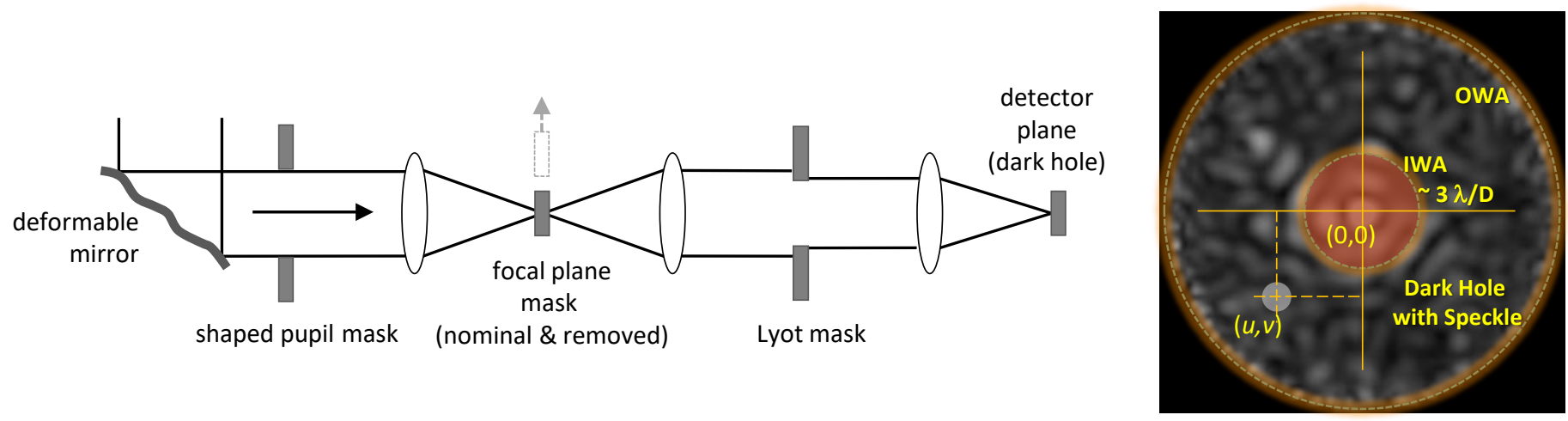
- Most important measure of coronagraph performance is contrast

- Contrast is defined as the fraction of star's light that leaks into the planet location $(u, v)$ relative to the light arriving at $(u, v)$ if the star were at the planet's location $(u, v)$.
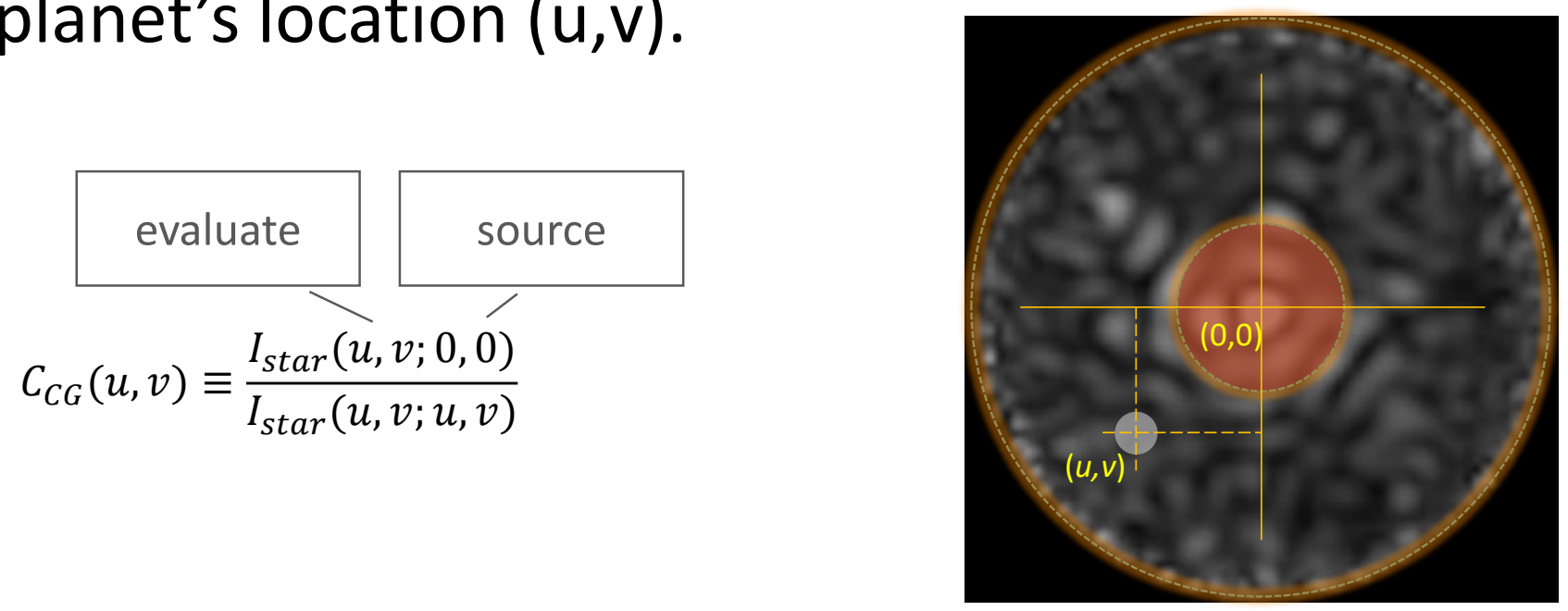

- Another important attribute is throughput.

- Core throughput is the fraction of the entering light from a planet that ends up in the planet's point spread function (PSF) "core" 
- Core throughput is a normalized encircled energy as a function of off-axis angle. It is the fraction of photons incident on the collecting aperture that end up within the half-max contour of the image plane PSF as a function of off-axis 'working' angle.

- Inner working angle is where throughput drops to $1 / 2$ of its max value

- Throughput drops because of Coronagraph vignetting near IWA.
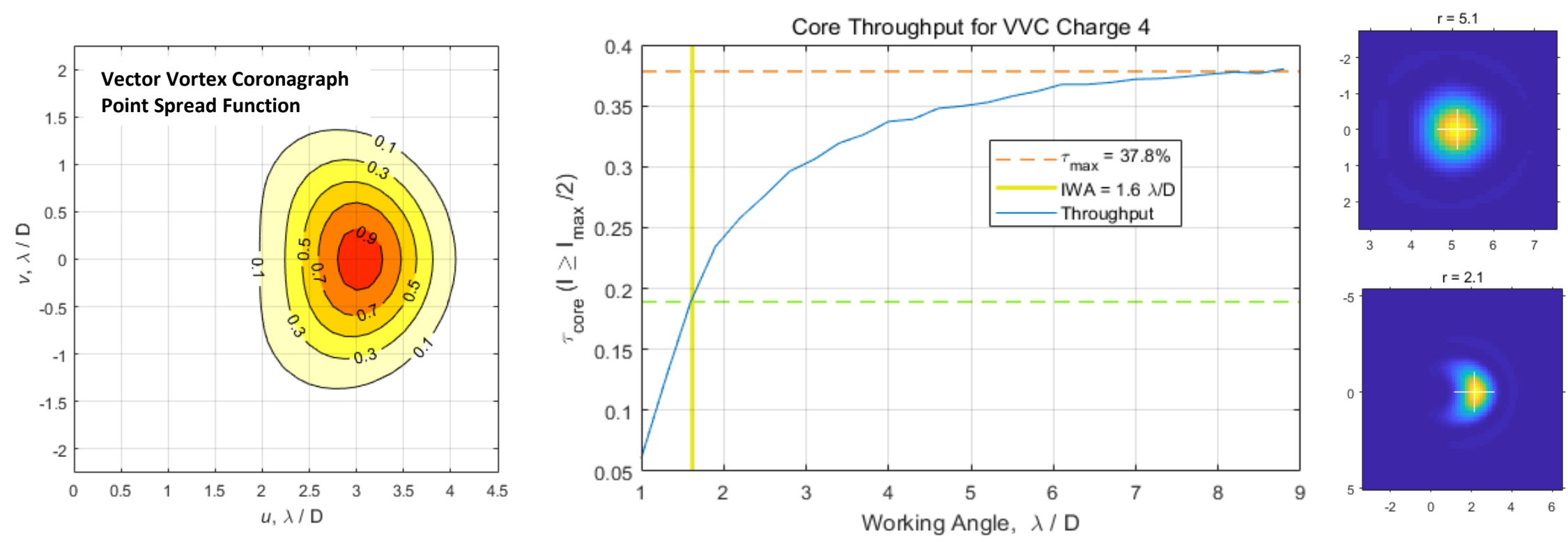
Performance of 4 telescope/coronagraph architectures studied.

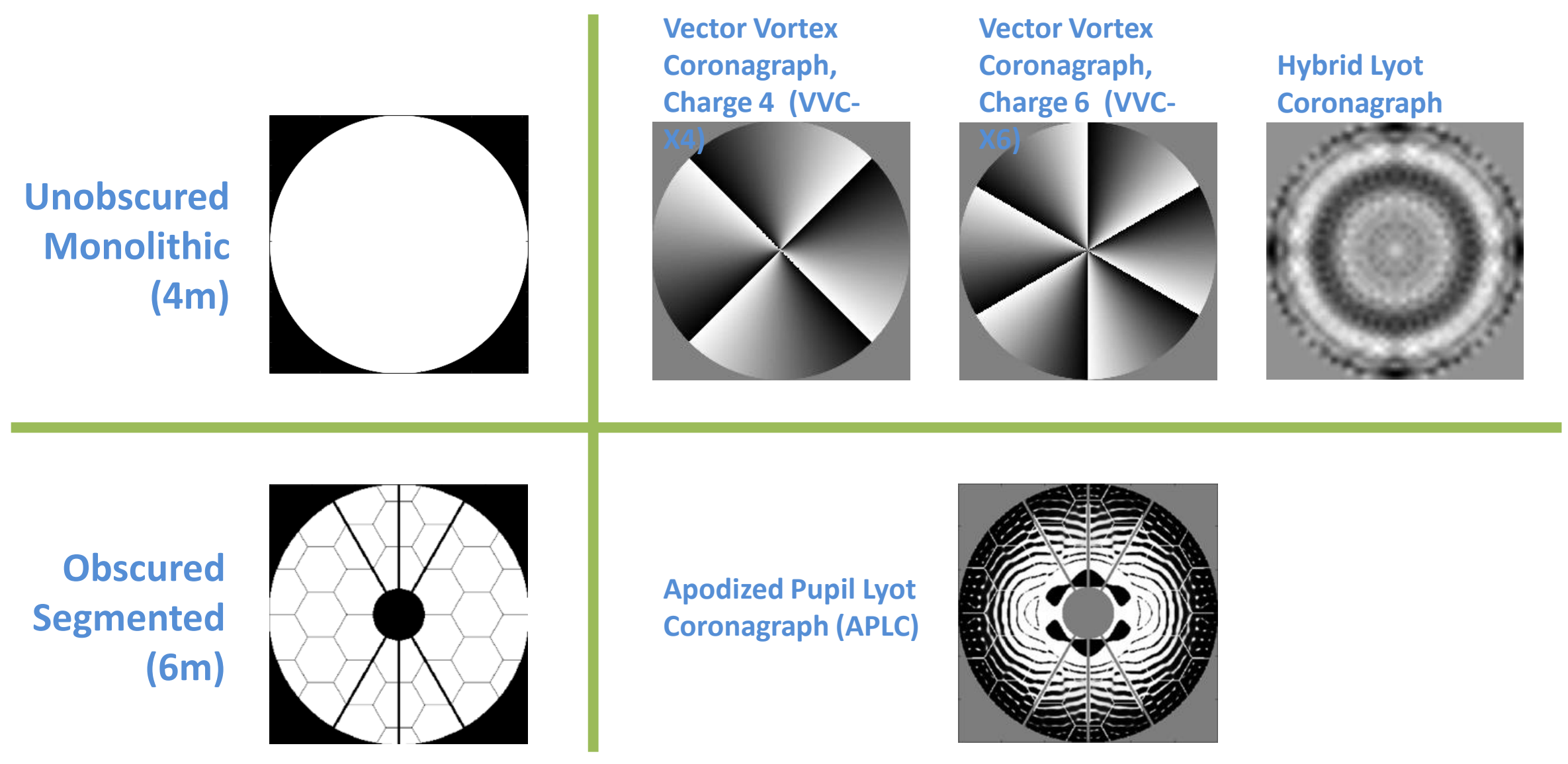




\section{Core Throughput Comparison}

- Comparison of core throughput versus separation angle for the four cases considered in this study.

- The separation for an Exo-Earth at 10 pc (100 mas) is indicated with the vertical line.

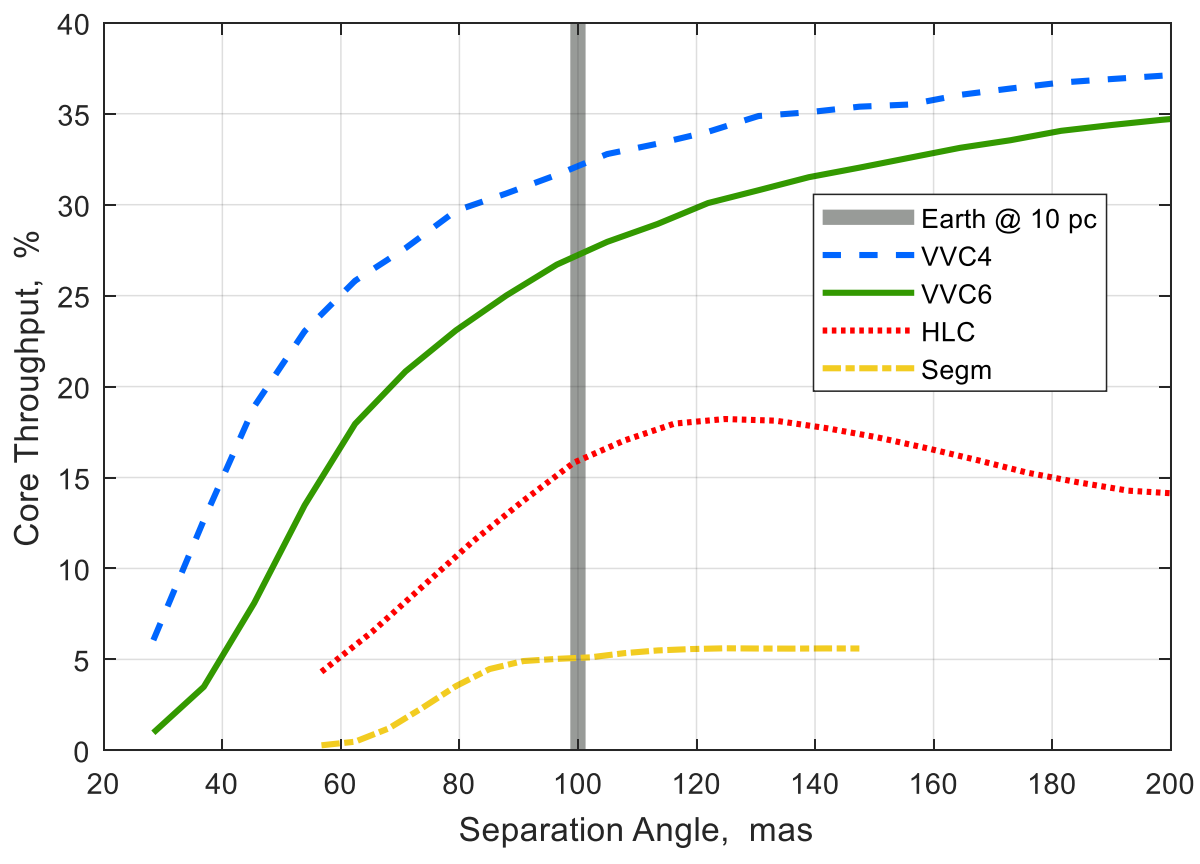

Note: $6 \mathrm{~m}$ aperture has aprox $2 \mathrm{X}$ more collecting area than $4 \mathrm{~m}$ aperture. Thus 'comparative' throughput @100 mas would be $10 \%$. 


\section{Imaging requires that planet signal has an adequate SNR $(R)$.}

There are two measurement error categories

Random Errors $\left(\sigma_{r}\right)$

Shot noise from signal and background sources (e.g. Zodi)

detector noise

Systematic Errors $\left(\sigma_{s}\right)$

Optical System Fixed Errors

Optical System Drift - Mechanical \& Thermal

residual speckle with a $\lambda / D$

spaced grid superimposed on top

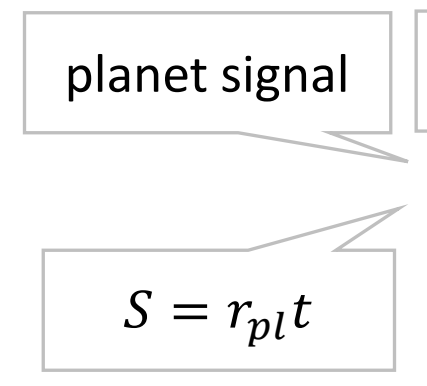

$$
\begin{aligned}
& \text { meas. noise } \\
& S / N=\mathrm{R} \\
& \quad N=\sqrt{\sigma_{r}^{2}+\sigma_{s}^{2}}
\end{aligned}
$$

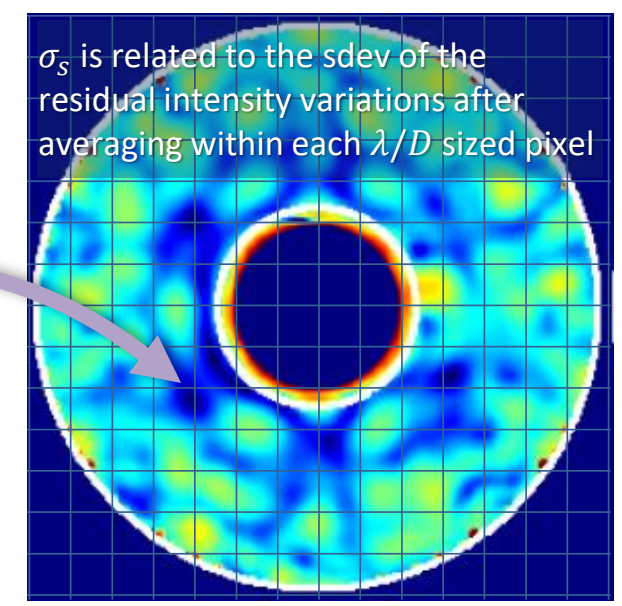

In this image there is no planet 


\section{Correcting Optical System Fixed Errors}

- Coronagraph requires 'flat' wavefront, but real systems have errors.

- Deformable Mirror can correct errors over a given spatial frequency range (base on its actuators) to create dark-hole.

- Imperfect Correction results in residual contrast systematic noise.

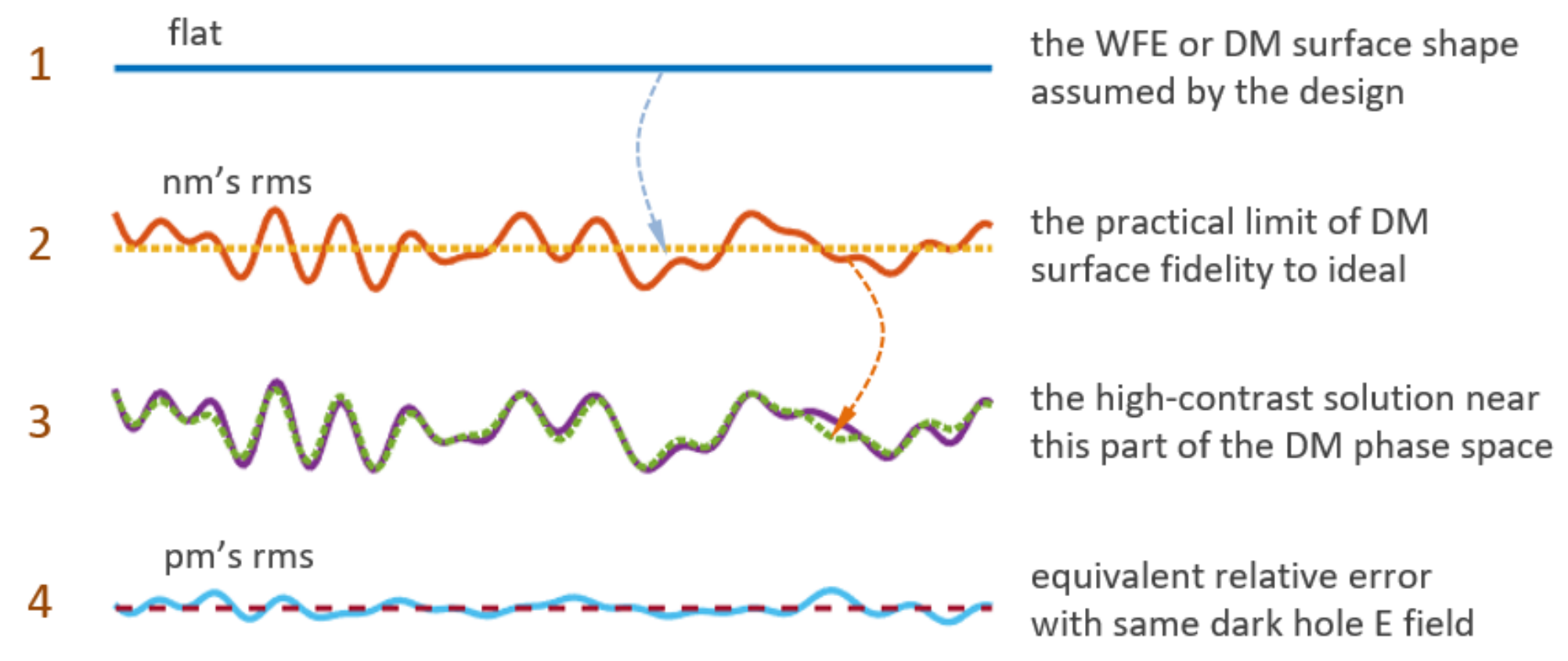




\section{Speckle Subtraction and Stability}

- One way to reduce residual speck noise is Speckle Subtraction via Reference Differential Imaging (RDI)

- Calibrate Dark-Hole Speckles on Reference Star

- Subtract Speckles from Target Star signal.

- Requires that Telescope is Stable over Slew

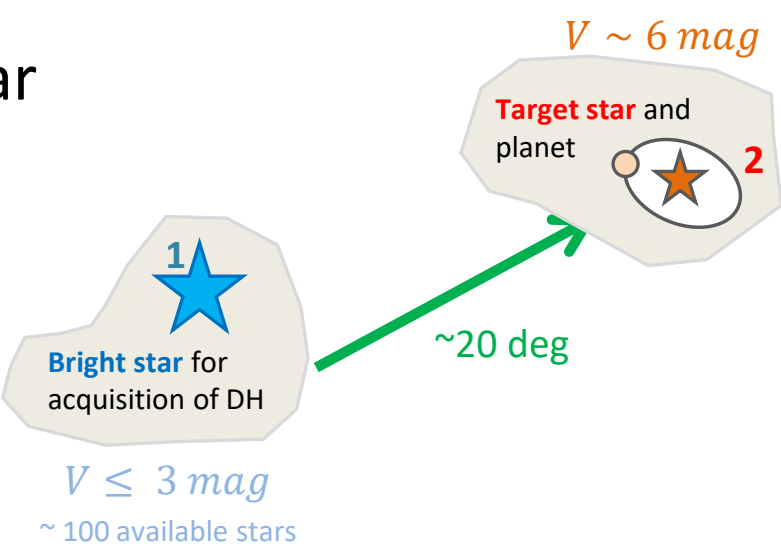

- Any Telescope Perturbation caused by slew appears in cross term.

$$
C \propto|E+\Delta E|^{2} \begin{aligned}
\begin{array}{l}
\text { existing field } \\
\text { (static field) }
\end{array} & \text { perturbation } \\
\text { (instability) } & \text { Cross term }
\end{aligned}
$$

- Instability is amplified by the existing $\mathrm{E}$ field in the cross term 


\section{Time to SNR and Contributions to Error}

$\mathrm{SNR}=7$

- To detect Exo-Earth with SNR = 7, Noise must be $<30$ ppt.

- Time to Detect depends on Noise.

- Random Noise is reduced by longer integration time

- But systematic noise (e.g. residual speckle) increases with time

- Science Integration time depends on initial residual speckle noise (WFE) and WFE growth with time.

- If Speckle Noise increases too fast, then need to recalibrate dark-hole.
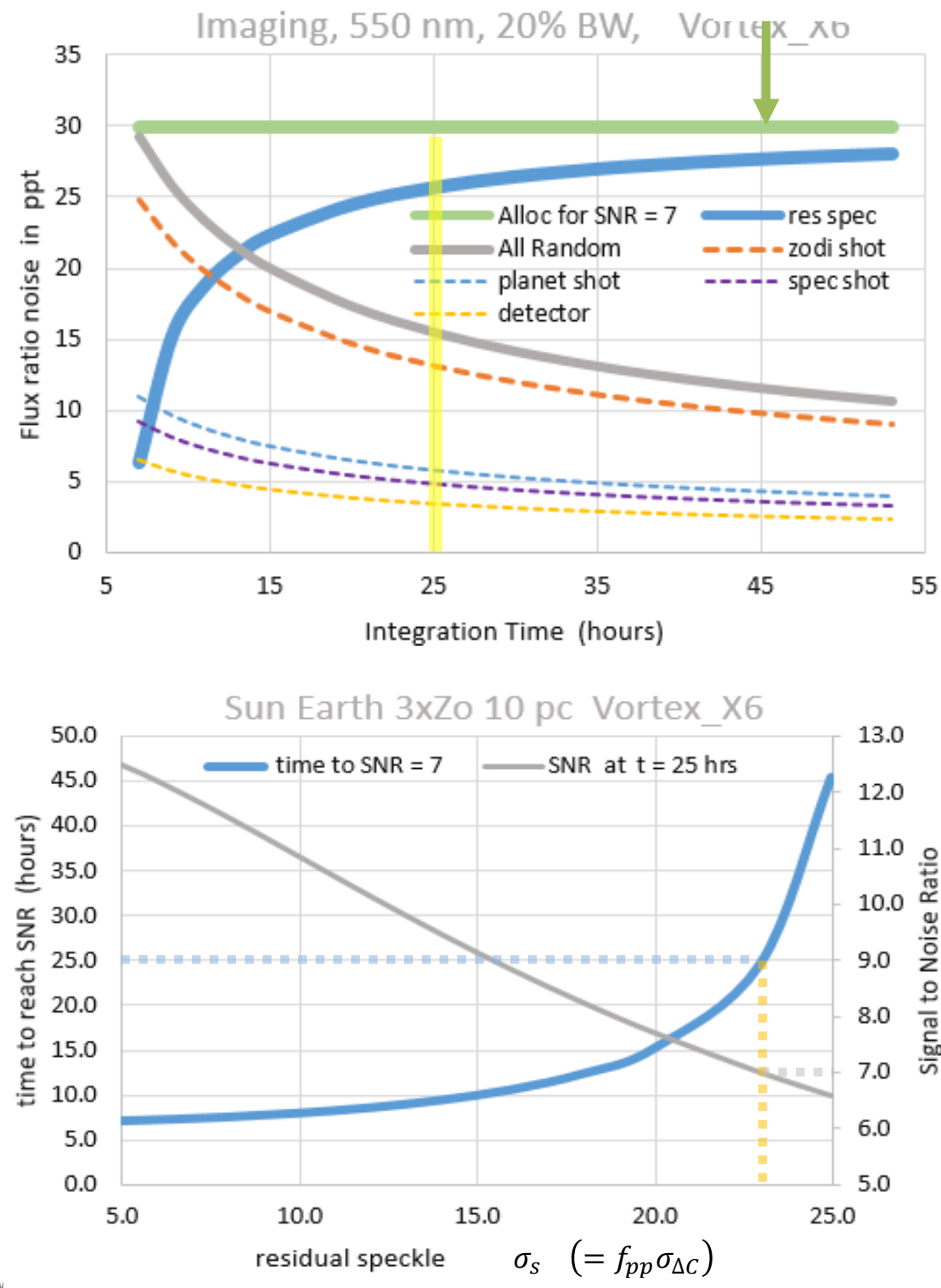


\section{Contrast Instability Error Budget}

For a given desired integration time create an Error Budget for the Telescope Contrast Instability.

- Start with Planet Flux Ratio and desired SNR = 7 to get Flux Ratio Noise

- Allocate noise between Random and Systematic

- Apply Post Processing Factors

- Contrast Instability is what must be achieved by the optical system.

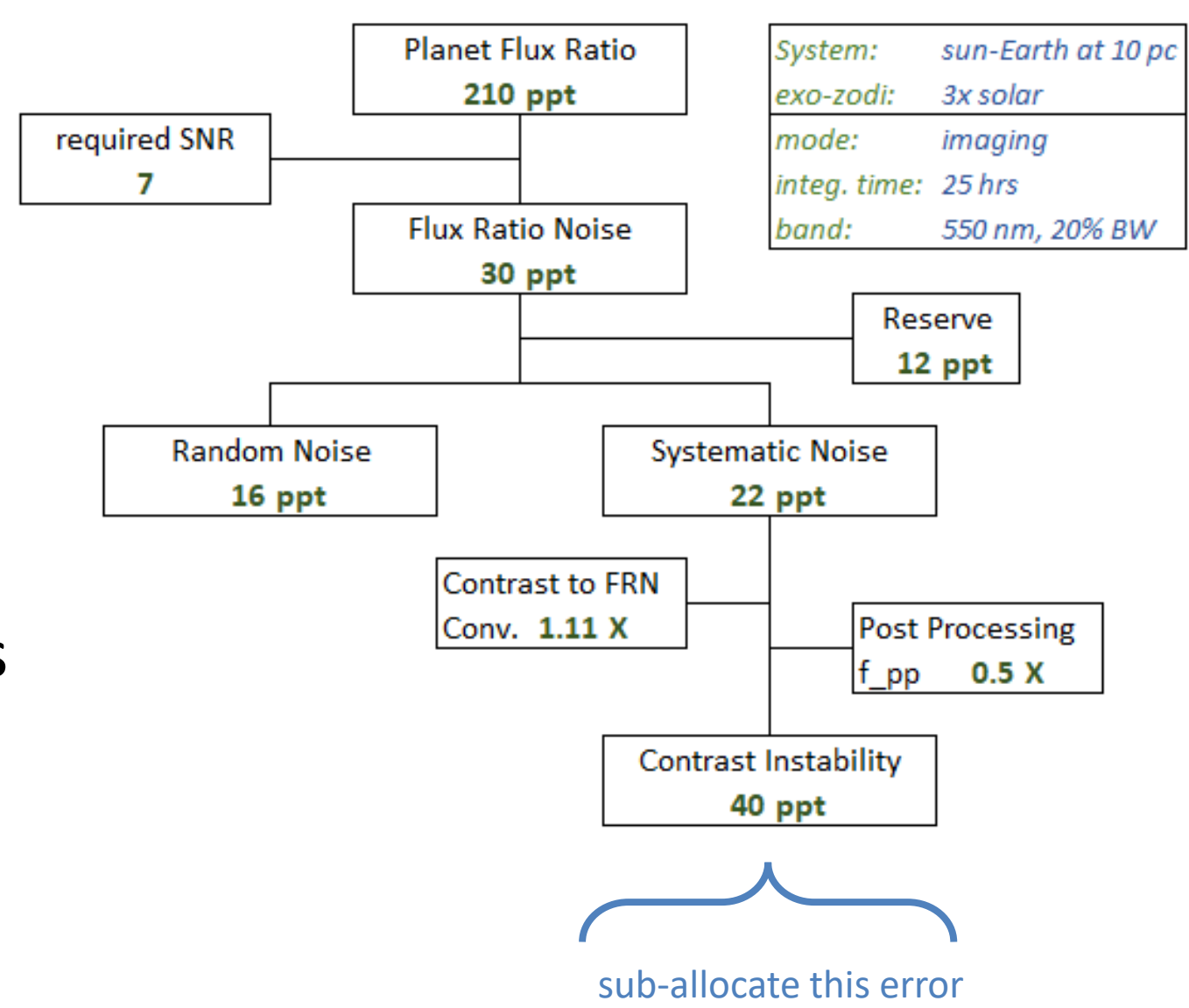




\section{Allocating Contrast Residual and Instability}

- How much star light gets scattered into a given speckle angular separation depends upon the amplitude of the wavefront error of a given spatial frequency (i.e. per the grating equation)

- Because WFE typically decreases with spatial frequency (PSD), residual speckle error sensitivity decreases with angular separation from star (requires higher spatial frequency error to scatter light)

- Contrast Instability must be allocated by Spatial Error Tolerance

$$
\begin{gathered}
\text { allocation } \\
\qquad \epsilon_{i}=\left(\frac{\partial \epsilon}{\partial x_{i}}\right)^{\text {tolerance }} \cdot \delta x_{i} \\
\text { sensitivity }
\end{gathered}
$$

- A convenient tolerance allocation is Zernike polynomials.

- Each Zernike polynomial WFE has a different Contrast Sensitivity. 
- Assume that $10 \mathrm{ppt}$ Contrast Instability is allocated to Trefoil

- Left shows residual speckle for 10 pm (PV) of trefoil WFE added between the reference and target star observations (VVC-6 case).

- Right shows Contrast Instability as a function of Trefoil WFE amplitude versus radial distance from star of integrated annular region

- Pink-shaded region shows radial distance where Contrast Instability allocation of $10 \mathrm{ppt}$ is exceeded for given Trefoil error.

- To see an Exo-Earth at 3.5 $\lambda / D$, Trefoil cannot exceed $\sim 12.5$ pm PV.
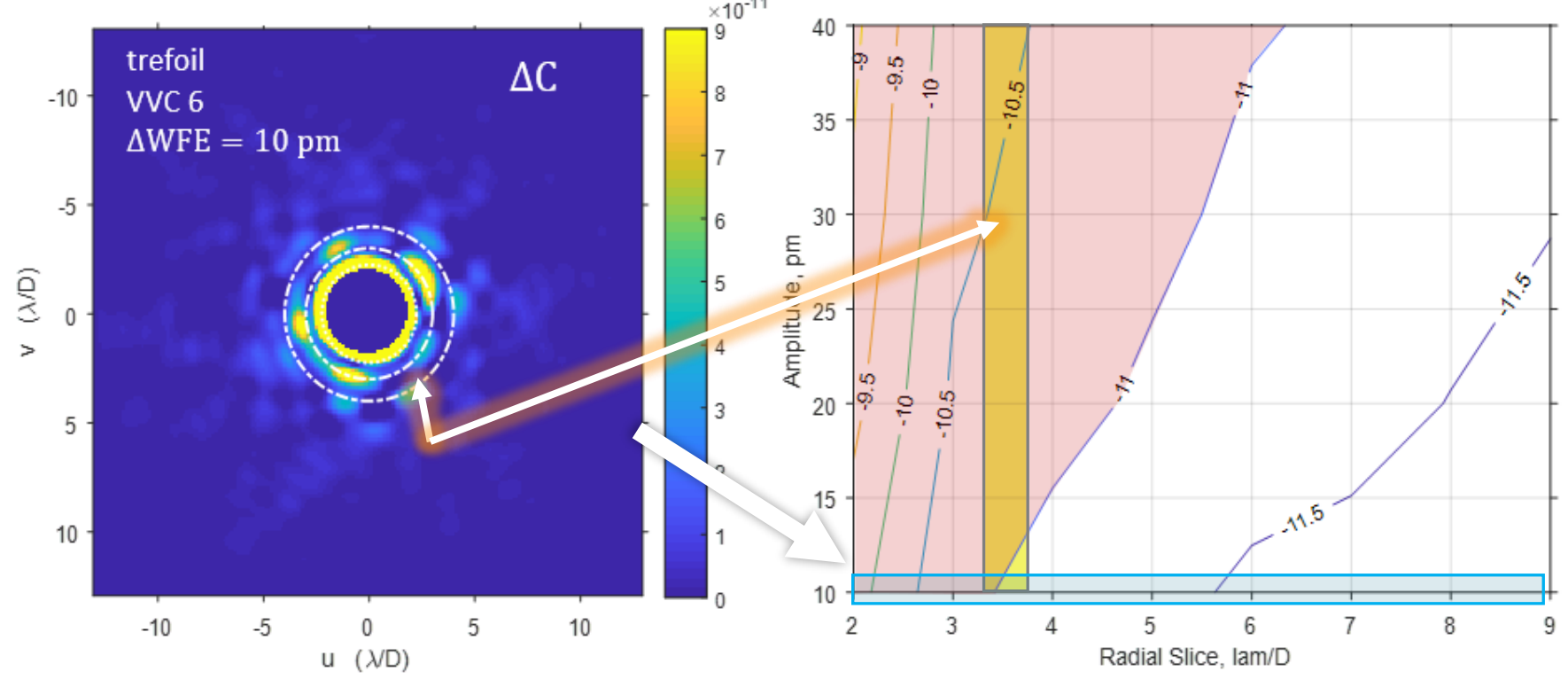
Plots show Contrast Sensitivity (for each case studied) to various Systematic WFE Changes at radial slice separation from Star for an Exo-Earth at $10 \mathrm{pc}$ observed at $550 \mathrm{~nm}$ (center).

- Vertical Line is Instrument $10^{-10}$ Raw Contrast needed to see Exoplanet

- Horizontal 10-11 Delta-Contrast Line is typical allocation per Zernike

- VVC X4 and X6 are insensitive to some low-order errors.

- Obscured segmented system is extremely sensitive to errors.
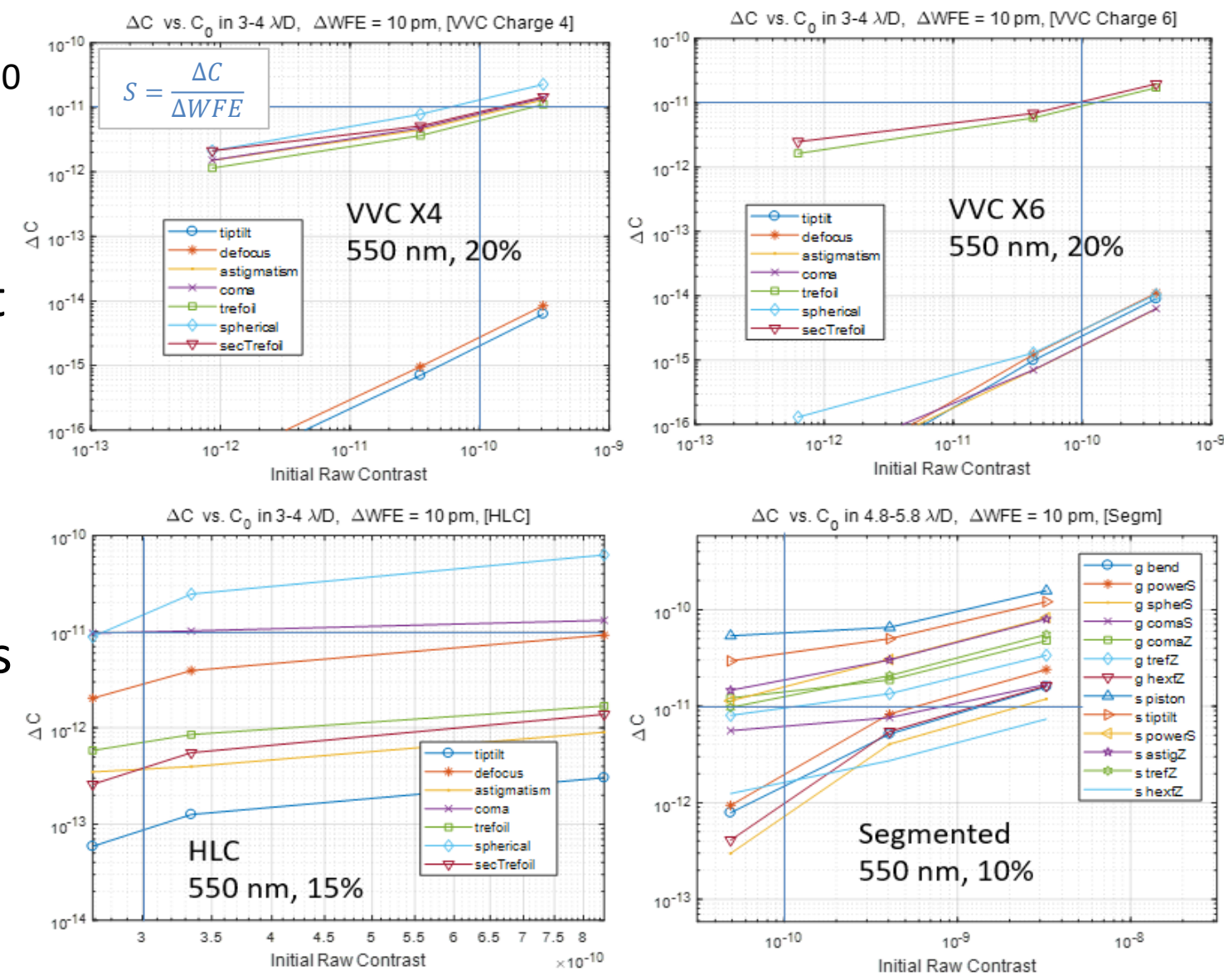


\section{Invert the Sensitivity Plots to determine WFE Allocations per Error}

\section{(Note: these are consistent with our previously published numerical simulation results)}

\begin{tabular}{|c|c|c|c|c|c|c|c|}
\hline$(\mathrm{CO}=100 \mathrm{ppt})$ & tiptilt & defocus & astigmatism & coma & trefoil & spherical & secTrefoil \\
\hline Sensitivities (ppt/pm) & 0.00024 & 0.00029 & 0.00017 & 0.00017 & 0.90 & 0.00029 & 1.04 \\
\hline Allocations (ppt) & 1.52 & 1.52 & 1.52 & 1.52 & 27.5 & 1.52 & 28.9 \\
\hline Tolerances (pm) & 6361 & 5170 & 9095 & 9026 & 30 & 5196 & 28 \\
\hline$(\mathrm{CO}=100 \mathrm{ppt})$ & tiptilt & defocus & astigmatism & coma & trefoil & spherical & secTrefoil \\
\hline Sensitivities (ppt/pm) & 0.00020 & 0.00027 & 0.77 & 0.82 & 0.64 & 1.35 & 0.86 \\
\hline Allocations (ppt) & 1.10 & 1.10 & 16.9 & 18.0 & 14.0 & 20.8 & 18.8 \\
\hline Tolerances (pm) & 5427 & 3996 & 22 & 22 & 22 & 15 & 22 \\
\hline$(\mathrm{CO}=300 \mathrm{ppt})$ & tiptilt & defocus & astigmatism & coma & trefoil & spherical & secTrefoil \\
\hline Sensitivities (ppt/pm) & 0.0095 & 0.305 & 0.037 & 0.990 & 0.073 & 1.738 & 0.042 \\
\hline Allocations (ppt) & 1.4 & 8.8 & 1.4 & 27.5 & 2.1 & 27.5 & 1.4 \\
\hline Tolerances (pm) & 153 & 29 & 39 & 28 & 29 & 16 & 35 \\
\hline
\end{tabular}

peak to valley

\begin{tabular}{|c|c|c|c|c|c|c|c|c|c|c|c|c|c|}
\hline$(\mathrm{CO}=100 \mathrm{ppt})$ & g_bend & g_powers & g_spherS & g_comaS & g_comaZ & g_trefZ & g_hexfz & s_piston & s_tiptilt & s_powers & s_astigZ & s_trefZ & s_hexfz \\
\hline Sensitivities (ppt/pm) & 0.15 & 0.21 & 0.090 & 0.59 & 1.32 & 0.89 & 0.12 & 5.53 & 3.26 & 1.44 & 1.71 & 1.14 & 0.15 \\
\hline Allocations (ppt) & 2.3 & 3.3 & 1.4 & 9.1 & 14.7 & 13.8 & 1.9 & 14.7 & 14.7 & 14.7 & 14.7 & 14.7 & 2.3 \\
\hline
\end{tabular}




\section{Summary and Conclusions}

- Exoplanet Science is hard. It requires that the telescope and coronagraph be designed as an integrated system.

- We describe a rigorous systems engineering methodology for deriving telescope performance specifications from coronagraph performance based on a raw contrast stability error budget.

- To illustrate the methodology, we apply it to four different architectures:

1. 4-m Off-Axis Unobscured Monolithic Circular Aperture with VVC-4 Coronagraph

2. 4-m Off-Axis Unobscured Monolithic Circular Aperture with VVC-6 Coronagraph

3. 4-m Off-Axis Unobscured Monolithic Circular Aperture with HLC Coronagraph

4. 6-m On-Axis Obscured Hex Segmented Aperture with APLC Coronagraph

- HabEx Baseline (4-m Monolith - VVC-6) has the best performance.

- Architecture 4 (6-m Segmented - APLC) has the worst total performance. 